\title{
Randomised controlled double blind study of role of recombinant erythropoietin in the prevention of chronic lung disease
}

Gill Griffiths, Ranjit Lall, Sue Chatfield, Andrew Short, Peter Mackay, Paula Williamson, Julia Brown, Malcolm I Levene

\begin{abstract}
Aim-To evaluate the role of recombinant human erythropoietin (R-HuEpo) in reducing iron infusion, which may exacerbate free radical damage, leading to chronic lung disease.

Methods-A multicentre, randomised, placebo controlled, double blind study was carried out in four neonatal intensive care units in Yorkshire. Infants were randomly allocated and received either R-HuEpo $(480 \mathrm{U} / \mathrm{kg} / \mathrm{wk})$ or placebo by twice weekly subcutaneous injection. The primary outcome measure was the number of days on respiratory support and a secondary outcome the number of blood transfusions required.
\end{abstract}

Results-Forty two very low birthweight (VLBW) infants were randomly allocated. There was little difference in the need for respiratory support one month after randomisation, but subsequently there was a trend towards a reduction in the proportion requiring respiratory support in the R-HuEpo group (difference at three months $-0.50,95 \%$ confidence interval $-1.00,0.17)$. During stay in hospital, the median number of blood transfusions was lower for infants in the R-HuEpo group (difference in medians $-2,95 \% \mathrm{CI}-4,0)$. The study was stopped early because of failure to recruit babies at the expected rate.

Conclusions-R-HuEpo seems to reduce the number of days in oxygen for ill VLBW infants. These data could be used to construct a larger multicentre study to evaluate this effect further.

(Arch Dis Child 1997;76:F190-F192)

Keywords: human erythropoietin; respiratory support; blood transfusions; chronic lung disease

Idiopathic respiratory distress syndrome (IRDS) is a common and important condition affecting premature infants. With the introduction of exogenous surfactant the mortality rate of IRDS has fallen by about $40 \%,{ }^{1}$ but there does not seem to have been any reduction in the proportion of surviving infants with chronic lung disease. ${ }^{1}$ This means that the number of infants surviving with prolonged additional oxygen requirements is increasing. This is very important both in terms of the morbidity of these infants and cost:benefit ratio, as keeping infants in hospital because of their need for additional oxygen is very expensive.

Oxygen toxicity is a factor in the development of chronic lung disease and is mediated through the release of oxygen free radicals. Ferrous iron accelerates the reaction between superoxide and hydrogen peroxide with production of hydroxyl radicals (the Haber-Weiss reaction). After birth the neonate becomes progressively depleted in iron and this may be an important protective mechanism against oxygen generated tissue injury. ${ }^{2}$ Premature infants undergoing intensive care are frequently given small volumes of donor blood to correct anaemia which may fuel the HaberWeiss reaction with the production of hydroxyl radicals and consequent lung injury. Raised serum ferritin concentrations (suggesting a greater availability of iron because of blood transfusion or haemolysis) are significantly higher in babies who develop chronic lung disease than in those who do not develop it. (Russell GAB, Cooke RWI. Iron associated antioxidants in complications of prematurity. Abstract G117. British Paediatric Association Meeting 1992). A recent overview of human recombinant erythropoietin (R-HuEpo) has shown a reduction in the need for blood transfusion in premature newborn infants. ${ }^{3}$

We hypothesised that if a therapeutic strategy could be devised to reduce the need for blood transfusion of free iron in high risk babies then the risk of chronic lung disease would be reduced. We report here a multicentre, randomised, placebo-controlled double blind study designed to evaluate the effect of $\mathrm{R}-\mathrm{HuEpo}$ on the requirement for supplemental oxygen.

\section{Methods}

Infants were recruited from four Yorkshire neonatal units between June 1993 and December 1994. Infants were enrolled in the study if they fulfilled all the following criteria: gestational age $\leqslant 32$ weeks and/or birthweight $\leqslant 1500 \mathrm{~g}$; requirement for mechanical ventilation and/or supplemental oxygen at birth; no severe renal, hepatic or coagulation disorders or major congenital malformation. If they still required mechanical ventilation/oxygen on day 7-14 after birth, and informed and signed parental consent had been given, these infants were randomly allocated to receive either R-HuEpo (Eprex; Cilag UK) 480 U/kg/week or placebo ( $4 \%$ human serum albumin). The first 
Table 1 Pre-treatment clinical assessments for 42 randomised infants

\begin{tabular}{lll}
\hline & R-HuEpo & Placebo \\
\hline Mean (SD): & $27(2)$ & $26(2)$ \\
Gestational age (weeks) & $28(2)$ & $28(2)$ \\
Postmenstrual age (weeks) & $940.5(194.2)$ & $923.1(194.4)$ \\
Birthweight (g) & $246.8(18.8)$ & $247.6(22.9)$ \\
Head circumference (mm) & $150.7(13.2)$ & $149.6(11.6)$ \\
Baseline heart rate (per min) & $28.3(7.0)$ & $27.3(5.6)$ \\
Lowest mean arterial BP (mm Hg) & & \\
No of infants (\%): & $12(57)$ & $17(81)$ \\
Respiratory support & $1(5)$ & 0 \\
$\quad$ IPPV & $8(38)$ & $4(19)$ \\
$\quad$ CPAP & $2(0-5)$ & $2(1-8)$ \\
$\quad \mathrm{O}_{2}$ alone & $29(0-73)$ & $32(10-94)$ \\
Median (range): & & \\
Number of blood transfusions & & \\
Volume: weight ratio of blood transfusion (m1/kg) & &
\end{tabular}

CPAP: continuous positive airway pressure.

injection was given on the day of randomisation, and then twice weekly until the infant either (a) reached 40 weeks postmenstrual age, (b) was transferred to another hospital (not in the study), (c) did not require any mechanical ventilation/oxygen for a period of more than a week, (d) had local adverse reactions/ complications related to the trial solution, or (e) had PCV $>60 \%$.

Stratified randomisation was used, accounting for participating centres, gestational age, and multiple births. Blinding was maintained throughout the study.

The primary endpoint of the study was the number of days the infant spent in mechanical ventilation/oxygen after randomisation. The secondary endpoints were (a) the number of blood transfusions, (b) the volume:weight ratio of the blood transfused, and (c) the incidence of chronic lung disease.

The requirement for additional oxygen was determined by transcutaneous oxygen saturation monitoring. Additional oxygen was no longer deemed necessary when (a) $\mathrm{SaO}_{2}$ was maintained $>93 \%$ in air when awake and (b)when there were no episodes of desaturation $(<90 \%)$ for $>1$ minute during sleep. Infants were transfused if they were ventilated and/or oxygen dependent with a haemoglobin of < $120 \mathrm{~g} / \mathrm{l}$, had clinically symptomatic anaemia, or were asymptomatic with a haemoglobin of $<70$ $\mathrm{g} / \mathrm{l}$. All infants in the study were to be treated

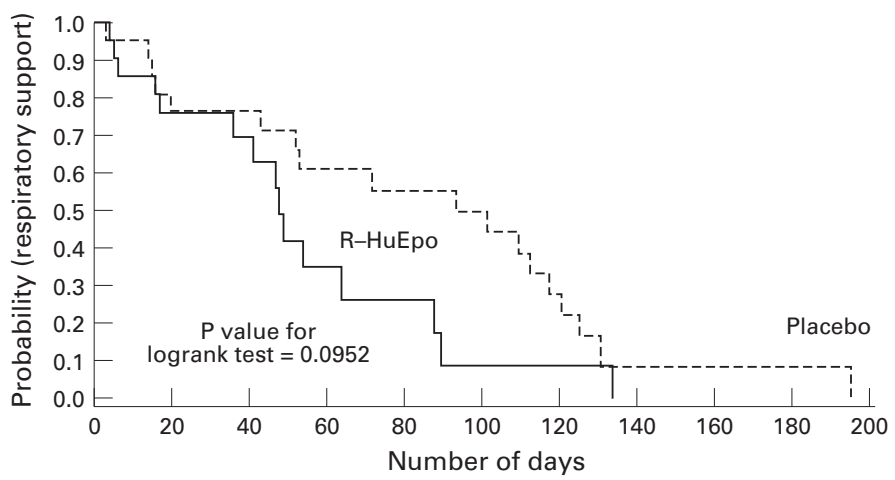

Number at risk

$\begin{array}{rrrrrrrrrrr}\text { Placebo 21 } & 16 & 15 & 12 & 10 & 9 & 5 & 1 & 1 & 1 & 0 \\ \text { R-HuEpo 21 } & 14 & 11 & 4 & 3 & 1 & 1 & 0 & 0 & 0 & 0\end{array}$

Figure 1 Kaplan-Meier curves showing the probabilities of receiving ventilatory support and/or supplemental oxygen. with oral iron $(3.0 \mathrm{mg} / \mathrm{kg} /$ day $)$ and folic acid $(0.5 \mathrm{mg} /$ day $)$ from four weeks after birth.

\section{STATISTICAL METHODS}

It was calculated that a total of 210 infants would be required (after accounting for withdrawals) to detect a difference of at least seven days in the average duration of respiratory support beyond the first week of life, with $80 \%$ power and a significance level of $5 \%{ }^{4}$ Duration of respiratory support was assumed to be exponentially distributed (suggested by retrospective data from one centre). However, this sample size was not achieved as a result of slower than expected accrual due to low parental consent rates and non-participation of a fifth centre. For these reasons the independent data monitoring committee decided that the trial should be stopped and the results used to plan a further study. Due to the early termination and the resultant small sample size, the results have been summarised by confidence intervals (CIs) only.

There was some evidence that in this study the duration of respiratory support was not exponentially distributed (Lagrange multiplier $\left.\chi^{2} \mathrm{P}=0.02\right)$. The distribution was estimated using the Kaplan-Meier method. ${ }^{56}$ The infants who died were censored in this analysis. A sensitivity analysis was also performed to assess the impact of deaths, by setting the duration of respiratory support for all infants who died equal to the maximum recorded. The number and volume:weight ratio of blood transfusions required were summarised between treatments using the difference in medians and $95 \%$ CIs. ${ }^{6}$ Chronic lung disease (CLD) was defined by an oxygen requirement at 36 weeks postmenstrual age, and was summarised using the difference in proportions and $95 \%$ CIs. All analyses were carried out on an intention-to-treat basis.

\section{Results}

Forty three infants were randomised. One infant who received treatment was ineligible and was subsequently withdrawn and excluded from the analysis. The trial treatment (placebo) was discontinued in a second infant after parental consent was withdrawn, but this infant was included in the analysis. The results are presented for 21 infants who received R-HuEpo and 21 who received placebo. Table 1 shows the characteristics of the two treatment groups before randomisation. These are broadly similar, although the placebo group may have had more severe respiratory illness, as suggested by the higher proportion of infants in intermittent positive pressure ventilation.

The duration of mechanical ventilation and/or supplemental oxygen is shown in fig 1 . The difference between R-HuEpo and placebo in the likelihood of the need for respiratory support at 1 and 3 months was -0.01 (95\% CI $-0.31,0.30)$ and $-0.50(95 \% \mathrm{CI}-1.00,0.17)$, respectively, in favour of R-HuEpo. The number of infants remaining in the study at 3 months was small, however. When the sensitivity analysis was carried out to account for deaths, the trend was still in favour of R-HuEpo. CLD occurred in $7 / 21$ (33.3\%) 
infants of the R-HuEpo group, compared with $12 / 21(57.1 \%)$ of the placebo group (difference in proportion $-0.24,95 \% \mathrm{CI}-53,5.4)$.

From randomisation until discharge home, infants given R-HuEpo received fewer blood transfusions (difference in medians -2, 95\% CI $-4,0)$ and had a lower volume:weight ratio $(\mathrm{ml} / \mathrm{kg})$ of blood transfused (difference in medians $-31,95 \% \mathrm{CI}-56,4)$.

Nine infants died during the study, six receiving $\mathrm{R}-\mathrm{HuEpo}$ and three in the control group. Three infants in the R-HuEpo group died from septicaemia, two from chronic lung disease, and one as a result of extreme prematurity. All three deaths in the placebo group were due to extreme prematurity. All infants who were alive at discharge were followed up to 6 months of age, and there were no additional deaths in either group. There was a total of 41 different types of adverse events, with infection (positive blood cultures), pneumonia, and patent ductus arteriosus being the most common.

\section{Discussion}

Although the number of infants remaining in the study at later times was small, the results for respiratory support showed a trend in favour of $\mathrm{R}-\mathrm{HuEpo}$. This is plausible on the basis of our hypothesis and supported by the reduction in the number of blood transfusions given to the babies in the R-HuEpo group together with a reduction in volume:weight ratio of blood transfused. Alternative reasons for the trend must be considered, and at randomisation there were fewer infants requiring mechanical ventilation in the group allocated R-HuEpo. This suggests that the placebo group had more severe respiratory illness which may explain some of the differences. No differences were found in the proportion of babies requiring respiratory support one month after randomisation. This is likely to have been due to the fact that in these very immature infants most required supplemental oxygen at the end of the first month after randomisation.

At 90 days $50 \%$ of babies required supplemental oxygen in the placebo group. Although we studied relatively few babies, we believe this represents the expected value for a group of high risk very immature infants. Half this proportion required mechanical ventilation and/or additional oxygen in the R-HuEpo treated group. This agrees with a recently published report on the role of erythropoietin on inhibiting lipid peroxidation and reducing the severity of alveolar damage in prematurely born rabbits exposed to high concentrations of oxygen.

This study was designed to investigate the role of R-HuEpo in the treatment of sick babies who required either supplemental oxygen or respiratory support at the end of the first week of life. The existing data concerning the effects of R-HuEpo on very small and sick infants are few. Most of the available information has been collected on relatively well and relatively large infants. In our study the median gestational age was 26 weeks, while in a recent overview of $\mathrm{R}-\mathrm{HuEpo}^{3}$ the average gestational age of randomised infants was less than 28 weeks in only four of 13 studies. In one study of R-HuEpo in infants with bronchopulmonary dysplasia the age when R-HuEpo treatment was started was 99 days. $^{8}$

Although the overall mortality rate in our study was relatively low $(21 \%)$ despite the high risk group, we were disappointed to note that there were more deaths in the R-HuEpo group. We cannot explain this, but in view of the small numbers involved, this could be a chance finding. A recent overview of $\mathrm{R}-\mathrm{HuEpo}$ treatment in premature infants reported no excess of deaths in the treatment group. ${ }^{3}$ Nevertheless, a data monitoring committee should oversee any future study with respect to unexplained deaths.

The trend suggested by this small study leads us to propose a large multicentre randomised controlled study to investigate this observation further. A number of points have been learnt from this study which will give more precision to the planning of a further study. The recruitment rate into the study was disappointing; we found that only $38 \%$ of eligible babies were randomised, with $27 \%$ of parents declining to take part. These figures will permit a more accurate calculation of the number of neonatal admissions required to recruit sufficient high risk babies.

Although we found a reduction in the need for blood transfusion, the dose of R-HuEpo used in our study was relatively low. There is now evidence that the most immature infants are more resistant to the effects of R-HuEpo and require a higher weekly dose. ${ }^{9}$ This must be considered further when planning a large multicentre study.

GG was funded by the Yorkshire Regional Health Authority

1 Soll RF, McQueen MC. Respiratory distress syndrome. In: Sinclair JC, Bracken MB, eds. Effective care of the newborn infant. Oxford: Oxford University Press, 1992:325-55

2 Sullivan JL. Iron, plasma antioxidants and the 'oxygen radical disease of prematurity. Am $\mathcal{f}$ Dis Child 1988;142: 1341-4.

3 Williamson P, Griffiths G, Norfolk D, Levene M. Blood transfusion and human recombinant erythropoietin in premature newborn infants. Arch Dis Child 1996;75:F65F8.

4 Machin D, Campbell M. Statistical tables for the design of clinical trials. Oxford: Blackwell Scientific Publications, 1987:132-9.

5 Peto R, Pike MC, Armitage P, Breslow NE, Cox DR, Howard SV, et al. Design and analysis of randomised clinical trials requiring prolonged observation of each patient. II. Analysis and examples. Br f Cancer 1977;35:1-39.

6 Gardner MJ, Altman DG. Statistics with confidence. London: British Medical Journal, 1989

7 Bany-Mohammed FM, Slivka S, Hallman M. Recombinant human erythropoietin: Possible role as an antioxidant in premature rabbits. Pediatr Res 1996;40:381-7.

8 Ohls RK, Hunter DD, Christensen RD. A randomized, double-blind, placebo-controlled trial of recombinant erythropoietin in treatment of the anemia of bronchopulmonary dysplasia. F Pediatr 1993;123:996-1000.

9 Shannon K. Recombinant erythropoietin in anaemia of prematurity: five years later. Pediatrics 1993;92:614-7. 